\title{
FORMULATION AND EVALUATION OF TOBRAMYCIN HYDROCHLORIDE ERODIBLE OCULAR INSERT
}

\author{
PATIL JA* AND PATIL RB \\ DCS's A. R. A. College of Pharmacy, Nagaon, Dhule \\ *Corresponding Author: Jayashri A. Patil: E Mail: jayshreeapatil@gmail.com
}

Received $19^{\text {th }}$ April 2021; Revised $20^{\text {th }}$ June 2021; Accepted $29^{\text {th }}$ July 2021; Available online $1^{\text {st }}$ Oct. 2021

https://doi.org/10.31032/IJBPAS/2021/10.10.1011

\begin{abstract}
Tobramycin is an aminoglycoside antibiotic used for treating various types of bacterial infections. The conventional liquid ophthalmic delivery systems exhibit short pre-corneal residence time and the relative impermeability to the cornea which leads to poor ocular bioavailability. Ocular inserts films are the most logical delivery systems that are aimed to remain for a long period of time in the front of eye. Hence aim of present study was to prepare Tobramycin hydrochloride erodible ocular insert by solvent casting method using Gelatin and Glycerin. Ocular inserts were evaluated for physicochemical parameters such as uniformity of thickness, uniformity of weight, drug content, water absorption test (Swelling index), surface $\mathrm{pH}$ and in-vitro diffusion study. Prepared ocular inserts were cross linked with $\mathrm{N}, \mathrm{N}^{1}$ - Bisacylamide (BIS) for getting sustained release of drug through ocular insert. Results of various parameters of non cross linked and cross linked ocular inserts showed that; solvent casting method is suitable for formulation of erodible ocular insert using gelatin and glycerin. Cross linked ocular insert showed sustained release of Tobramycin $\mathrm{HCl}$ as compared to non cross linked ocular insert. Result showed that, erodible inserts of Tobramycin $\mathrm{HCl}$ would be able to offer benefits such as increasing residence time, prolonging drag release, reducing frequency of administration, and thereby may help to improve patient compliance.
\end{abstract}

Keywords: Tobramycin hydrochloride, Erodible Ocular Insert, Gelatin, Glycerin, Invitro diffusion study 


\section{INTRODUCTION}

Ocular infections are mostly chronic in nature and are characterized by inflammation of the cornea, conjunctiva, and eyelids. Common ophthalmic infections include conjunctivitis, blepharitis, keratitis, keratoconjunctivitis, and sty. The management of anterior segment ocular infection and inflammation requires prompt diagnosis and treatment to avoid any serious visual complications [1]. Bacterial infections are commonly treated using a combination of antibiotic and steroidal drugs. The antibiotic reduces the infection, while the corticosteroid treats the inflammation associated with the infection [2]. For ophthalmic use, a number of new preparations have been developed in order not to prolong the contact time of the vehicle on the ocular surface, but to reduce the drug elimination speed. Most ocular treatments like eye drops and suspensions call the topical administration of ophthalmic drugs to the tissues around the ocular cavity. These dosage forms are easy to instil but have the inherent drawback that the majority of the medication in them is immediately diluted. Poor bioavailability of drugs from ocular dosage forms is mainly due to the tear production, non-productive absorption transient residence time, and impermeability of corneal epithelium. Successful results have been recorded for inserts [3]. It also offers accurate dosing to overcome the side effects of pulsed dosing by conventional systems, increases the ocular bio availability of drugs by prolonging the corneal contact time, to circumvent the protective barriers like drainage, lacrimation and conjuctival absorption [4].

Tobramycin is a water soluble aminoglycoside antibiotic produced by the fungus Streptomyces tenebrarius and used in a variety of pharmaceutical applications including ophthalmic solutions, suspensions and ointments; inhalation solutions and intravenous administration [5, 6]. Tobramycin is an aminoglycoside antibiotic used for treating various types of bacterial infections. Tobramycin irreversibly binds to the bacterial $30 \mathrm{~S}$ and 50S ribosomal subunits, preventing the formation of $70 \mathrm{~S}$ complex, which eventually inhibits mRNA translation, leading to cell death [7]. TOBRADEX ${ }^{\circledR}$ is a sterile, multiple dose antibiotic and steroid combination marketed formulation for topical ophthalmic use. The disadvantages of current marketed formulations are burning, stinging, irritation, itching to the eyes due to dispersed particles $[8,9]$.

Hence present study was aimed to prepare Tobramycin hydrochloride erodible ocular insert by solvent casting method with the rationale of prolonging the contact of the 
drug with ocular tissue and facilitating a constant concentration of the drug.

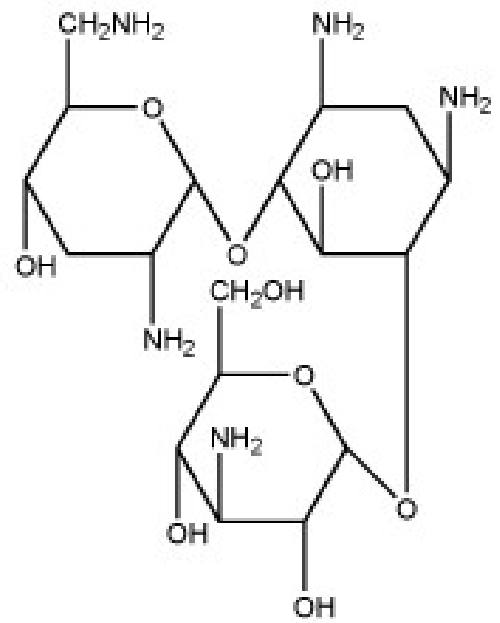

Figure 1: Chemical structure of Tobramycin MATERIALS AND METHODS

\section{MATERIALS:}

Tobramycin was obtained as a generous gift from Sun Pharmaceuticals, Vadodara, India. Gelatin and Glycerin were purchased from SD Fine chemicals, Mumbai, India. All other chemicals used were of analytical grade.

\section{METHODS:}

Formulation of Tobramycin Erodible Ocular Insert

The ocular inserts were prepared using solvent casting method. The nine batches (TF1 to TF9) of ocular inserts were prepared using different concentration of gelatin $(16 \%, 18 \%, 20 \%)$ and glycerin $(70 \%, 50 \%, 40 \%)$. The required quantity of gelatin and glycerin were weighed and dissolved in three quarter quantity of water and the mixture was heated at $60^{\circ} \mathrm{C}$ on a water bath until the entire gelatin was dissolved. The weighed amount of tobramycin and the remaining water were added and stirred on magnetic stirrer to get uniform dispersion. After complete mixing the casting solution $(5 \mathrm{ml})$ was poured on petridish containing mercury as substratum. The petridish was cooled at $10^{\circ} \mathrm{C}$ by placing on ice, until the films were gelled. The gelled films were taken out from ice and allowed to dry at room temperature for $72 \mathrm{~h}$. The petri dish was covered by an inverted funnel with cotton plug to prevent aerial contamination of the films during the drying period. The dried films thus obtained were cut to the required size and stored till use $[\mathbf{1 0}, \mathbf{1 1}]$.

Table 1: Compositions of different batches of Tobramycin HCl ocular insert

\begin{tabular}{|c|c|c|c|}
\hline Batch & Tobramycin HCl & Gelatin (\%) & Glycerin (\%) \\
\hline TF1 & 0.3 & 12 & 80 \\
\hline TF2 & 0.3 & 12 & 60 \\
\hline TF3 & 0.3 & 12 & 40 \\
\hline TF4 & 0.3 & 14 & 80 \\
\hline TF5 & 0.3 & 14 & 60 \\
\hline TF6 & 0.3 & 14 & 40 \\
\hline TF7 & 0.3 & 16 & 80 \\
\hline TF8 & 0.3 & 16 & 60 \\
\hline TF9 & 0.3 & 16 & 40 \\
\hline
\end{tabular}


Evaluation of Tobramycin $\mathrm{HCl}$ Ocular Inserts:

\section{Thickness}

The thickness of the insert was determined using a vernier caliper at five separate points of each insert. From each formulation, five randomly selected inserts were tested for their thickness [12].

\section{Uniformity of weight}

From each batch, five inserts were weighed individually using digital balance. The mean weight of the insert was noted. The percent deviation was calculated from individual weight of the inserts [13].

\section{Drug content}

Five ocular inserts were taken from each batch and dissolved in $25 \mathrm{ml}$ of isotonic phosphate buffer $\mathrm{pH} 7.4$ into volumetric flask. One $\mathrm{ml}$ of the above solution was withdrawn and the absorbance was measured by UV-VIS spectrophotometer at $285.6 \mathrm{~nm}$ after suitable dilution [14].

\section{Folding endurance}

Five films of each formulation of size $(20 \mathrm{x}$ $20 \mathrm{~mm}$ ) were cut by using sharp blade. Folding endurance was determined by repeatedly folding a small strip of the film at the same place till it broke. The number of times the film could be folded at the same place without breaking gave the value of folding endurance. A mean of five reading was recorded [15].

$\%$ Moisture absorption
The percentage moisture absorption test was carried out to check physical stability or integrity of the film at humid condition. The five inserts were taken from each formulation. Individual inserts were weighed and placed in a desiccator containing saturated solution of aluminium chloride and $84 \%$ humidity was maintained [16]. After three days, the inserts were reweighed. The $\%$ moisture absorption was calculated using the formula:

$\%$ Moisture absorption $=\{($ Final weight - Initial Weight)/Final Weight $\}$ * 100

Surface pH

The inserts were allowed to swell in closed petridish at room temperature for $30 \mathrm{~min}$ in $0.1 \mathrm{ml}$ of bidistilled water. "The swollen device was removed and placed under digital $\mathrm{pH}$ meter to determine the surface $\mathrm{pH}$ [17].

\section{In-vitro diffusion study}

The in-vitro diffusion of Tobramycin $\mathrm{HCl}$ from the different formulations was studied using the classical standard cylindrical tube fabricated in the laboratory a simple modification of glass tube of $15 \mathrm{~mm}$ internal diameter and $100 \mathrm{~mm}$ height. The commercial semi-permeable membrane cellophane, presoaked overnight in the freshly prepared dissolution medium (isotonic phosphate buffer $\mathrm{pH}$ 7.4), was tied to one end of open cylinder (open at both the sides), which acted as a donor compartment. An ocular insert was placed 
inside this compartment. The diffusion cell membrane acted as corneal epithelium. The entire surface of the membrane was in contact with the receptor compartment comprising of $25 \mathrm{ml}$ of isotonic phosphate buffer $(\mathrm{pH} \mathrm{7.4)}$ in a $100 \mathrm{ml}$ beaker. The content of receptor compartment was stirred continuously using a magnetic stirrer and temperature was maintained at $37^{\circ} \pm 0.5^{\circ} \mathrm{C}$. At specific time intervals, 1 $\mathrm{ml}$ aliquot of sample was withdrawn from the receptor compartment and replaced with fresh buffer solution. The aliquot was analyzed for the drug content using UVVIS spectrophotometer at $300 \mathrm{NM}$ after appropriate dilutions against reference using isotonic phosphate buffer $\mathrm{pH} 7.4$ as blank $[18,19]$.

\section{Cross Linking of Tobramycin $\mathrm{HCl}$}

\section{Ocular Inserts}

Out of nine formulations (TF1 to TF9), two formulations $\mathrm{TF} 4(14 \% \mathrm{w} / \mathrm{v}$ gelatin and $80 \% \quad \mathrm{w} / \mathrm{w}$ glycerin) and TF7 (16\% $\mathrm{w} /$ vgelatin and $80 \% \mathrm{w} / \mathrm{w}$ glycerin) were selected and cross linked. The cross linking was done by dipping cut inserts $(8 \mathrm{~mm}$ diameter) in $10 \% \mathrm{w} / \mathrm{v}$ solution of $\mathrm{N}, \mathrm{N}^{1}$ Bisacylamide (BIS) in isopropyl alcohol (5 $\mathrm{ml}$ ) for four different time period of 15,30 , 45 and $60 \mathrm{~min}$. In order to remove excess unreacted BIS from the surface of inserts, the films were transferred to an aqueous sodium metabisulphite solution $(2 \%)$ and then immediately removed and placed in absolute alcohol bath. The formulations so prepared were designated as THF1, THF2, THF3, THF4, THF5, THF6, THF7 and THF8. The hardened inserts were kept in desiccators until use $[\mathbf{2 0}, \mathbf{2 1}]$. The compositions of Tobramycin ocular inserts are shown in Table 2.

\section{Evaluation of Cross linked Tobramycin HCl Ocular Inserts}

The cross linked ocular inserts were evaluated for uniformity of thickness, uniformity of weight, drug content, water absorption test (Swelling index), surface $\mathrm{pH}$ and in-vitro diffusion study as per procedure described for evaluation of Tobramycin $\mathrm{HCl}$ Ocular Inserts.

Table 2: Cross linking of Tobramycin HCl Ocular Inserts

\begin{tabular}{|c|c|c|c|}
\hline Batch & Hardening Time (min) & Gelatin (\%) & Glycerin (\%) \\
\hline THF1 & 15 & 14 & 80 \\
\hline THF2 & 30 & 14 & 80 \\
\hline THF3 & 45 & 14 & 80 \\
\hline THF4 & 60 & 14 & 80 \\
\hline THF5 & 15 & 16 & 80 \\
\hline THF6 & 30 & 16 & 80 \\
\hline THF7 & 45 & 16 & 80 \\
\hline THF8 & 60 & 16 & 80 \\
\hline
\end{tabular}


RESULT AND DISCUSSION

Evaluation of Tobramycin HCl Ocular Inserts

\section{Thickness:}

The thickness of formulations (TF1 to TF9) was found to be in range of $0.187 \mathrm{~mm}$ to $0.297 \mathrm{~mm}$ as shown in the Table 3. The uniformity in thickness of all formulations ensured that, solvent casting method is suitable for the preparation of ocular inserts.

\section{Weight variation (\% deviation):}

The $\%$ deviation in weight of all formulations was found to be in the range of 0.29 to 0.83 . The uniformity of the weights of the films indicated good distribution of the drug, gelatin and glycerin. It was found that the thickness and weight of the inserts increased with increasing total polymer concentration.

\section{Drug content:}

Drug content of all formulations was found to be in the range of 95.47 to $99.67 \%$. It showed that uniform distribution of drug in all formulations.

\section{Folding endurance:}

Folding endurance of all formulations was found to be in the range of 119 to 192 . The folding endurance test results indicated that the inserts would not break and maintain their integrity with general folding when applied. Results showed that, as concentration of plasticizer increases folding endurance also increases.
\% Moisture absorption:

$\%$ Moisture absorption of all batches was found to be in the range of 5.78 to 7.93 . Results showed that, as concentration of gelatin increases; percentage of moisture absorption also increases as polymers are more hydrophilic in nature. Moreover, at humid conditions increase in moisture absorption was observed without affecting physical appearance of ocular inserts.

\section{Surface pH:}

The $\mathrm{pH}$ of the gelatin solution is almost neutral (7.01). Surface $\mathrm{pH}$ of all batches was found to be in between 6.28 to 7.26. Results indicate that the prepared inserts would not alter the $\mathrm{pH}$ of the tear fluid in the eye. Hence there would not cause irritation in the eye.

\section{In-vitro diffusion study of Tobramycin HCl Ocular Inserts}

In-vitro diffusion of Tobramycin $\mathrm{HCl}$ from Non cross linked ocular insert (TF1 to TF9) was found to be 97.62 to $99.72 \%$ in 3 to 5 hours. Results showed that, as concentration of gelatin increases \% diffusion of drug from ocular insert decreases. Results also showed that, non cross linked ocular insert showed complete drug release within 5 hours. From evaluation of all nine batches formulation TF4 and TF7 was found to be satisfactory and hence further used for cross linking (Figure 2). 
Evaluation of Cross linked Tobramycin HCl Ocular Inserts

Thickness of cross linked Tobramycin $\mathrm{HCl}$ ocular inserts was found to be in the range of 0.242 to $0.297 \mathrm{~mm}$. The \% deviation of weight variation was found to be in the range of 0.28 to 0.84 . There was very little gain in weight and increase in thickness after cross-linking in the films. The films retained their integrity and appearance after cross-linking, but there was a considerable hardening of the film surface [10].

Drug content was found to be in the range of 96.45 to $99.77 \%$. Folding endurance was found to be in the range of 196 to 210 which is higher than non cross linked ocular insert. The reason behind the high folding endurance was high concentration of plasticizer i.e. $80 \% \mathrm{w} / \mathrm{w}$ used in the formulations resulted in higher flexibility of the films. \% Moisture absorption (swelling behaviour) was found to be in the range of 3.59 to $4.32 \%$. Swelling of the formulations significantly decreased on cross-linking. This indicates that the swelling of the film has considerably decreased because of surface treatment. As the cross-linking extent increased, the surface pores might have decreased, leading to significantly decreased water uptake. The $\mathrm{pH}$ of all cross linked ocular insert was found to be in the range of 6.58 to 7.42. This shows prepared inserts would not cause irritation in the eye (Table 4).

In-vitro diffusion of Tobramycin $\mathrm{HCl}$ from Cross linked ocular insert (THF1 to THF8) was found to be 88.79 to $99.76 \%$ in 12 hours. Batch THF4 and THF8 showed maximum drug release i.e. 99.76 and 98.16 $\%$ in 12 hours respectively. As compared to non cross linked ocular insert batch (TF4 and TF7); cross linked ocular insert showed extended release of Tobramycin $\mathrm{HCl}$ up to 12 hours. It is well known that gelatin has an excellent property of increasing viscosity as its concentration increases. Furthermore, gelatin swells upon imbibition of water molecules in it and retards/ slows down the movement of drug molecules outside of the matrices. The sustaining action of the polymeric films could be explained by the cross-linking mechanism of gelatin and $\mathrm{N}, \mathrm{N}^{1}$ Bisacylamide (Figure 3).

Table 3: Evaluation of Tobramycin HCl Ocular Inserts

\begin{tabular}{|c|c|c|c|c|c|c|}
\hline Batch & $\begin{array}{c}\text { Thickness } \\
\text { (mm) }\end{array}$ & $\begin{array}{c}\text { Weight variation } \\
\text { (\% deviation) }\end{array}$ & $\begin{array}{c}\text { Drug content } \\
(\%)\end{array}$ & $\begin{array}{c}\text { Folding } \\
\text { endurance }\end{array}$ & $\begin{array}{l}\text { \% Moisture } \\
\text { absorption }\end{array}$ & Surface pH \\
\hline TF1 & 0.187 & 0.36 & 96.85 & 156 & 5.78 & 6.28 \\
\hline TF2 & 0.179 & 0.47 & 98.36 & 135 & 6.02 & 6.54 \\
\hline TF3 & 0.193 & 0.39 & 95.47 & 119 & 5.89 & 6.69 \\
\hline TF4 & 0.238 & 0.29 & 99.67 & 178 & 6.96 & 7.12 \\
\hline TF5 & 0.263 & 0.63 & 98.62 & 169 & 7.31 & 7.26 \\
\hline TF6 & 0.273 & 0.83 & 97.94 & 136 & 6.89 & 7.14 \\
\hline TF7 & 0.286 & 0.34 & 98.95 & 192 & 7.66 & 7.23 \\
\hline TF8 & 0.292 & 0.68 & 97.52 & 184 & 7.58 & 6.98 \\
\hline TF9 & 0.297 & 0.76 & 98.71 & 178 & 7.93 & 7.37 \\
\hline
\end{tabular}




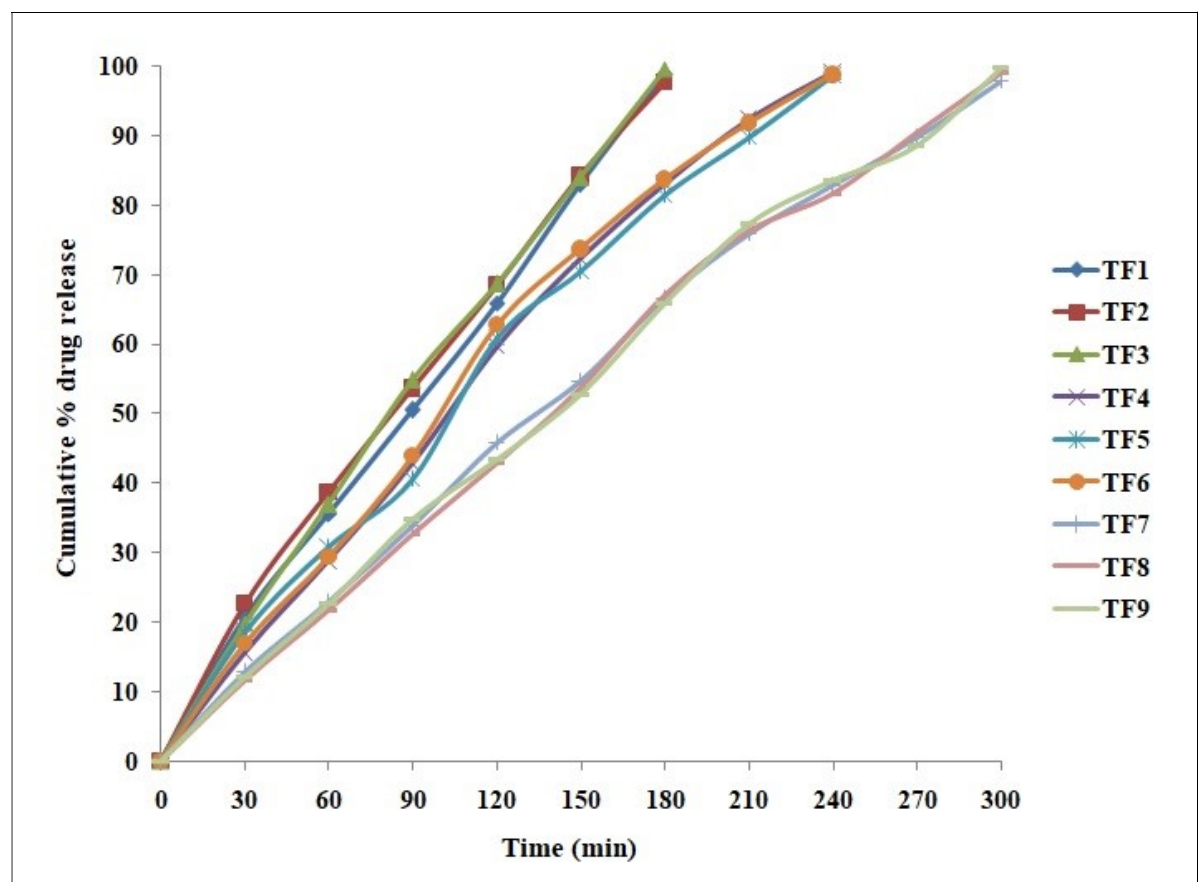

Figure 2: In-vitro diffusion of Tobramycin HCl from Non cross linked ocular insert

Table 4: Evaluation of Cross linked Tobramycin HCl Ocular Inserts

\begin{tabular}{|c|c|c|c|c|c|c|}
\hline Batch & $\begin{array}{c}\text { Thickness } \\
\text { (mm) }\end{array}$ & $\begin{array}{c}\text { Weight variation } \\
\text { (\% deviation) }\end{array}$ & $\begin{array}{c}\text { Drug } \\
\text { content }(\%)\end{array}$ & $\begin{array}{c}\text { Folding } \\
\text { endurance }\end{array}$ & $\begin{array}{l}\text { \% Moisture } \\
\text { absorption }\end{array}$ & Surface pH \\
\hline THF1 & 0.242 & 0.46 & 96.45 & 197 & 4.32 & 6.58 \\
\hline THF2 & 0.248 & 0.52 & 98.73 & 200 & 3.96 & 6.78 \\
\hline THF3 & 0.252 & 0.28 & 99.62 & 198 & 3.87 & 6.79 \\
\hline THF4 & 0.265 & 0.79 & 96.76 & 196 & 4.12 & 6.92 \\
\hline THF5 & 0.289 & 0.63 & 97.15 & 202 & 3.98 & 7.42 \\
\hline THF6 & 0.291 & 0.84 & 98.37 & 204 & 4.22 & 7.15 \\
\hline THF7 & 0.295 & 0.39 & 99.62 & 206 & 3.71 & 7.28 \\
\hline THF8 & 0.297 & 0.48 & 99.77 & 210 & 3.59 & 7.15 \\
\hline
\end{tabular}

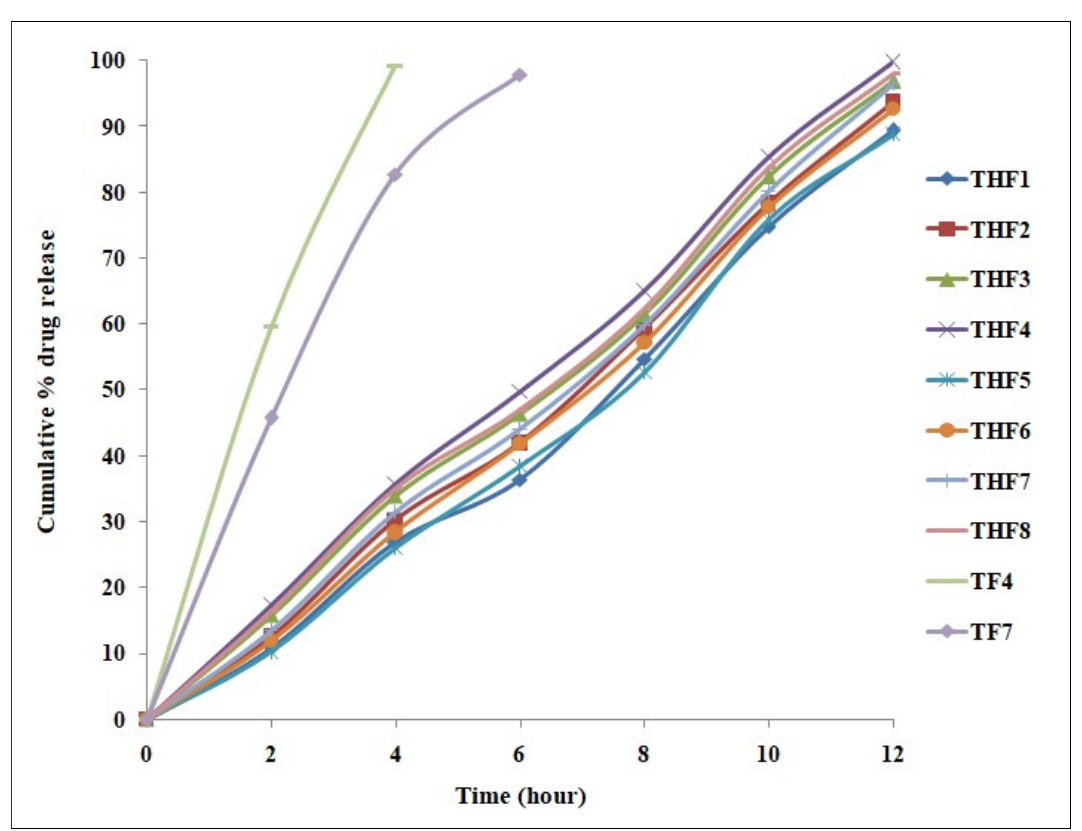

Figure 3: In-vitro diffusion of Tobramycin HCI from cross linked ocular insert (THF1 to THF8) and comparison with two non cross linked batches (TF4 and TF7) 


\section{CONCLUSION}

Erodible ocular insert of Tobramycin $\mathrm{HCl}$ was successfully prepared by solvent casting method using gelatin and glycerine and sustained effect can be obtained by cross linking of with $\mathrm{N}, \mathrm{N}^{1}$ - Bisacylamide. From results it can be concluded that, the films retained their integrity and appearance after cross-linking, but there was a considerable hardening of the film surface which will help in sustaining the release of drug from insert. The formulations hence fulfilled all the pharmaceutical parameters of ocular inserts and appear to be promising, would be able to offer benefits such as increasing residence time, prolonging drug release, reducing frequency of administration, and thereby may help to improve patient compliance.

\section{REFERENCES}

[1] Rinda Devi Bachu, Marina Stepanski, Rami M. Alzhrani, Rose Jung, and Sai H.S. Boddu. Development and Evaluation of a Novel Microemulsion of Dexamethasone and Tobramycin for Topical Ocular Administration. 2018; $1-13$.

[2] Boddu, S.H., Bonam, S.P., and Jung, R. Development and characterization of a ricinoleic acid poloxamer gel system for transdermal eyelid delivery. Drug. Dev. Ind. Pharm. 2015; 41: 605-612.
[3] Mishra D, Gilhotra R. Design and characterization of bioadhesive in-situ gelling ocular inserts of gatifloxacin sesquihydrate. Daru J. Pharm. Sci. $2008 ; 16: 1-8$.

[4] Gurtler F and Gurny R. Patent literature review of ophthalmic inserts. Drug Dev Ind Pharm. 1995; 21:1-18.

[5] Hanko VP, Rohrer JS. Determination of tobramycin and impurities using highperformance anion exchange chromatography with integrated pulsed amperometric detection. J Pharm Biomed Anal, 2006; 40 (4):1006-1012.

[6] Manyanga V, Elkady E, Hoogmartens J, Adams E. Improved reversed phase liquid chromatographic method with pulsed electrochemical detection for tobramycin in bulk and pharmaceutical formulation. J Pharm Anal, 2013; 3(3):161-167.

[7] Babic F., Venturi V., and MaravicVlahovicek G. Tobramycin at subinhibitory concentration inhibits the RhlI/R quorum sensing system in a Pseudomonas aeruginosa environmental isolate. BMC Infect. Dis. 10:1, 2010 .

[8] Nirav Patel, Vaishali Thakkar, Viral Metalia, Lalji Baldaniya, Tejal Gandhi \& Mukesh Gohel. Formulation and development of ophthalmic in situ gel for the treatment ocular inflammation and infection using application of 
quality by design concept. Drug

Development and Industrial Pharmacy. 2016; 42(9): 1406-1423.

[9] https://www.rxlist.com/tobradex-sideeffects-drug-center.htm

[10]Manish Mathurm, and Ritu Mehra Gilhotra. Glycerogelatin-based ocular inserts of aceclofenac: Physicochemical, drug release studies and efficacy against prostaglandin E2induced ocular inflammation. Drug Delivery, 2011; 18(1): 54-64.

[11] Manvi FV, et al. Development and evaluation of timolol maleate ocular inserts. Indian Drugs.1997; 34(5):264268.

[12]Rao V and Shyale S. Preparation and evaluation of ocular inserts containing norfloxacin. Turk J Med Sci. 2004; 34:239-246.

[13] Harishkumar SL, Balasubramaniam J, Thilek Kumar M, Pandit JK. In vitro characterization of physically reinforced ocular inserts of indomethacin. Acta Pharmaceutica Turcica 2004; 46:17-24.

[14]Mundada AS and Shrikhande BK. Design and evaluation of soluble ocular drug insert for controlled release of ciprofloxacin hydrochloride. Drug Development and Industrial Pharmacy 2006; 32: 443 - 448.

[15] Sreenivas SA, Hiremanth SP and Godbole AM. Ofloxacin ocular inserts:
Design, formulation and evaluation. IJPT 2006; 5:159-62.

[16]Balasubramaniam J, Srinatha A, Pandit JK, Gopalnath. In vitro microbiological evaluation of polyvinyl alcohol based ocular inserts of ciprofloxacin hydrochloride. Indian. J. Pharm. Sci. 2006; 68:626-630.

[17]Mukherjee P, Manvi FV, Sreenivas SA. Sulphacetamide sodium ocular inserts: Design, formulation and evaluation. Indian Drugs 2006; 43:705704.

[18] Vijayendra SM, Nanjawade BK. Development of sustained ocular drug delivery system for betaxolol. Indian. J. Pharm. Educ. Res. 2006; 40:165168.

[19]Ahuja M, Gupta A, Sharma SK. In vitro and In vivo Evaluation of gellan based ocular inserts of phenylephrine. Acta Pharmaceutica Scieneia 2007; 49: 55-63.

[20] Ashture Amar, Kankudte Ashish, Patil Ajaykumar, Joshi Anand. Formulation and evaluation of controlled release ocular inserts of betaxolol hydrochloride. IOSR Journal of Parmacy. 2012; 2(5): 34-38.

[21]Mundada AS, et al. Design and evaluation of soluble ocular drug inserts for controlled release of ciprofloxacin hydrochloride. Indian $\mathrm{J}$ Pharm Sci.2004; 570-577. 